\title{
Compaction Characterization and Model Prediction of Stabilized UnyegheResidual Soils, AkwaIbomState Nigeria
}

\author{
EssienUdo ${ }^{1}$, Ini $\mathrm{Umoh}^{2}$, Joseph Samuel ${ }^{3}$ \\ ${ }^{1,2,3}$ Dept. of Civil Engineering, University of Uyo, Nigeria
}

\begin{abstract}
The primary step in the construction of sub-base and base course materials is the identification and selection of suitable borrow pits. This is done by obtaining samples from economically feasible borrow pits and testing them in the laboratory. ${ }^{.}$The laboratory compaction with varying compactive effort viz: British Standard (BS) compaction with $2.5 \mathrm{~kg}$ rammer, repeated on 3 layers of 61 blows each, West African Standard (WAS) compaction with $4.5 \mathrm{~kg}$ rammer repeated on 5 layers of 25 blows each; and Heavy British Standard (HBS) compaction with $4.5 \mathrm{~kg}$ rammer repeated on 5 layers of 61 blows each on samples from locations 1 and 2 respectively were conducted. Focus was on Unyeghe residualsoils from two distinct borrow-pits stabilized with river sand and ordinaryPortland cement. In all cases, the rammers fell from a height of $450 \mathrm{~mm}$ above the top of mould. The unsoakedCBRvalues obtained with BS and WAS compactions, $34 \%$ and $46 \%$ respectively, were far below the recommended minimum of $35 \%$ and $80 \%$ for sub-base and base courses applications by FMW \& $H^{2}(1997)$ specification. The HBS compaction tends to simulate the actual field condition by limiting the air voids to about $5 \%$. An interesting feature observed is that the highest CBR and MDD values obtained, $(132 \%, 134 \%$ and $\left.2100 \mathrm{~kg} / \mathrm{m}^{3}, 2010 \mathrm{~kg} / \mathrm{m}^{3}\right)$ occurred at lower moisture contents $(7.6 \%, 9.4 \%)$ at both locations. On application of the BS and WAS compactive effort to OPC stabilized Unyeghe residual soil samples the soaked CBRand MDD values at optimal level $\left(124 \%, 132 \%\right.$ and $\left.2000 \mathrm{~kg} / \mathrm{m}^{3}, 2060 \mathrm{~kg} / \mathrm{m}^{3}\right)$, showed comparative improvement. This result could not be justified only by direct influence attributable to the stabilizing materials only. It could thus be concluded that both the California Bearing Ratio (CBR) and Maximum Dry Density (MDD) while not being inherent properties of the soil material, are predicated on the applied compactive effort. Hence, the more the soil material is compacted, the greater the value of cohesion and shearing resistance. Multiple nonlinear regressed models were developed for the purpose of prediction and optimization of Unyeghe residual soils with various stabilizing parameters.
\end{abstract}

Keywords: Compaction, Stabilization, Residual Soil, River Sand, Cement.

\section{Introduction}

A variety of mechanical equipment is used to compact soils in the field. Various types of rollers are being used in road construction. Each type of roller has special mechanical systems to effectively compact a particular type of soil. For example, a sheep-foot roller is generally used to compact fine-grained soils while a drum type roller is generally used to compact coarse-grained soils ${ }^{3} \mathrm{An}$ interesting question is "what type of roller is suitable for compacting a stabilized residual soil from Unyeghe?"The British Standard, West African Standard and Heavy British Standard compaction were deployed for this laboratory investigation. In order to carry out proper evaluation of design properties of stabilized residual soils a sound understanding of compaction characteristics required is of essence.Equally the optimum moisture content and the maximum dry density of soils are very important parameters for construction specifications of soil improvement by compaction. Specifications for earth structures usually call for a minimum of $95 \%$ of maximum dry unit weight. This level of compaction can be attained at two water contents; one, before the attainment of the maximum dry density, the other after attainment of the maximum dry density. Normal practice is to compact the soil at the lower water content value except for swelling [expansive] soils. Compaction increases the strength, lowers the compressibility, and reduces the permeability of a soil by rearranging its fabric. The soil fabric is forced into a denser configuration by the mechanical effort used in compaction. Compaction is therefore the most popular method or technique of improving soils structure especially the stabilized soil structure.

\subsection{Unyenghe Residual soils}

\section{Materials Selected}

Two soils samplesselected for this research were dug with shovels from two borrow-pits along Kilometer 2+250 Unyeghe-EsitEket road and Kilometer 9+400 Unyeghe-Stubb Creek access road. The soil samples were disturbed and at depths varying from 3.0 meters to 5.0 meters of the profile. The samples were excavated bearing in mind the variability of residual soil in its natural composition. Hence the soil samples were excavated both vertically and laterally and thoroughly blended. The samples were 
conveyed in two, $50 \mathrm{~kg}$ nylon bags, carefully tagged for identification purpose and transported to the Mothercat Limited, Materials Testing Laboratory at Uyo.

\subsection{River Sand}

This is one of the most abundant stabilizing materials within the coastal plains and tributaries of the Atlantic. The material was obtained from a tributary of the Cross river in Itu. The deleterious and silty substances were thoroughly removed by washing. The material was then air-dried before particle size gradation through sieve analysis. Sand plays a vital role in enhancing the bond in cementation reactions of soil mixing. It is found that grain size distribution provides a satisfactory skeleton, and the voids are filled with fine sand giving a compact and high load bearing capacity. From analysis the sand is observed to have a mean diameter $\mathrm{d}_{50}$ equal to $0.620 \mathrm{~mm}$ and effective diameter $\mathrm{d}_{10}$ of $0.300 \mathrm{~mm}$

\subsection{Cement}

The cement used in this research was the ordinary Portland cement ${ }^{4}$ (OPC). It was purchased from Ewet market in Uyo. This cement is the most widely used in the construction industry in Uyo, Akwalbom State. Ordinary Portland Cement particle is a heterogeneous substance, containing minute tri-calcium silicate $\left(\mathrm{C}_{3} \mathrm{~S}\right)$, di-calcium silicate $\left(\mathrm{C}_{2} \mathrm{~S}\right)$, tri-calcium aluminate $\left(\mathrm{C}_{3} \mathrm{~A}\right)$ and solid solution described as tetra calcium aluminoferrite $\left(\mathrm{C}_{4} \mathrm{~A}\right)$ [Lea, 1956]. When the pore water of the soil encounters with cement, hydration of the cement occurs rapidly and the major hydration (primary cementitous) produces hydrated calcium silicate $\left(\mathrm{C}_{2} \mathrm{SHx}, \mathrm{C}_{4} \mathrm{AHx}\right)$ and hydrated lime $\mathrm{Ca}(\mathrm{OH})_{2}$. [Bergado, et.al.1996]. In the case of residual soils addition of inorganic chemical such as cement has a two-fold effect on the soil which is acceleration and promotion of chemical bonding.

\subsection{Gradation Test}

\section{Preparation And Testing Ofsamples}

After air-drying the samples for three weeks the first step was to sieve through $20 \mathrm{~mm}$ diameter sieve and any particle retained was broken with rubber hammer or thrown away. With the aid of a riffle box the quantity of material needed or five hundred grams each of the soil samples were extracted and poured into sieve no.200 or $0.075 \mathrm{~mm}$ diameter sieve and thoroughly washed toremove all clayey materials finer than the $0.075 \mathrm{~mm}$ diameter. The particles retained were oven-dried, weighed and mechanically sieved in a shaker.

\subsection{Liquid Limit Test.}

The method adopted, utilized the Casagrande 5 apparatus. It must be noted that Arthur Casagrande made one of the most important contributions to geotechnical engineering; ordering and presenting clearly the existing differences between objectives for civil engineering soils classification and soil classification schemes intended for other purposes. The air-dried samples were quantified through a sample divider - the riffle box - and sieved through $425 \mu \mathrm{m}$ test sieve $50 \mathrm{~g}$ of material passing through this sieve was used for the liquid limit test. The sample was put in a flat glass plate, moisturized and thoroughly mixed with a spatula to a thick homogeneous paste. The paste was preserved in air-tight polythene sack for 24 hours to allow water permeate the entire sample, devoid of moisture evaporation. It was then put back into the glass plate and properly mixed for 15 minutes. Finally the paste was then put into the Casagrande liquid limit apparatus, grooved to $\mathrm{V}$-shape as per specification, to determine the number of blows that will be required to bring the two parts into contact. The range of blows varied from 10-15, 15-20, 21-30, and 31-40 and for various moisture contents.

\subsection{Plastic Limit Test}

This test determines the lowest moisture content at which the soil is plastic. About $60 \mathrm{~g}$ of samples passing the $425 \mu \mathrm{m}$ test sieve was moisturized and thoroughly mixed in the glass plate until it becomes homogeneous and plastic, enough to be shaped into a ball. The ball was then rolled between the palms of the hand, until the heat of hands dried the sample sufficiently for slight cracks to appear on its surface. It was then rolled 
continuously forward and backward in between the finger and glass plate until the pressure was sufficient to reduce the diameter of the thread to about $3 \mathrm{~mm}$. The procedure was repeated until the thread sheared (crumbled) both longitudinally and transversely.

\subsection{Compaction and Stabilization Tests.}

These were the main experiments conducted to study the response and behavior of Unyeghe residual soils on various levels of standard compactive efforts. Similar procedures were adopted on application of additives or stabilizers of various percentages to the dry unit weight of the air-dried samples. The stabilizers utilized include river sand and cement.

\subsection{Plain Mechanical Compaction}

This test was conducted to determine the mass of dry soil per cubic meter and the soil was compacted in a specified manner over a range of moisture contents, including that giving the maximum mass of dry soil per cubic meter. For each of the samples, the Heavy British Standard compaction test was conducted. The air-dried material was divided into five equal parts through a riffle box and weighed to $6000 \mathrm{~g}$ each. Each sample was poured into the mixing plate. A particular percentage of distilled water was poured into each plate and thoroughly mixed with a trowel. An interval of about 1 hour was allowed for the moisture to fully permeate the soil sample. The sample was thereafter divided into five equal parts, weighed and each was poured into the compaction mold, in five layers and compacted at 61 blows each using a $4.5 \mathrm{~kg}$ rammer falling over a height of $450 \mathrm{~mm}$ above the top of the mold. The blows were evenly distributed over the surface of each layer. The collar of the mold was then removed and the compacted sample weighed while the corresponding moisture content was noted. The procedure was repeated with different moisture contents until the weight of compacted sample was noted to be decreasing. With the optimum moisture content obtained from the WAS test, samples were prepared in the CBR mold and values for the plain mechanical compaction were read for both top and bottom at various depths of penetration.

\subsection{Stabilization Tests ${ }^{6}$.}

Different percentages of stabilizers varying from $10 \%, 20 \%, 30 \%, 40 \% .50 \%, 60 \%$ and $70 \%$ were added to air-dried samples 1 and 2 . Each of the test samples was thoroughly blended with a trowel, divided into five parts with the aid of a riffle box, moisturized and weighed. The percentages of residual soil ranged from $90 \%, 80 \%, 70 \%, 60 \%, 50 \%$, and $40 \%$ to $30 \%$ thus complementing the $100 \%$ per test specimen weighed at $6000 \mathrm{~g}$ each. Thereafter the comparative compaction testswere carried out to determine the OMC and MDD. Liquid limit and plastic limit tests were conducted on each of the samples.

\subsection{River Sand Stabilization Tests.}

River sand sufficient fines will fill the voids thus giving a compact and high load bearing capacity ${ }^{7}$. Samples ranging from $10 \%, 20 \%, 30 \%, 40 \%, 50 \%, 60 \%$ to $70 \%$ by weight of the air-dried residual soils were utilized in this stabilization tests. For each of the residual soil samples 1 and 2 different proportions of a $6000 \mathrm{~g}$ weight ranging from $90 \%, 80 \%, 70 \%, 60 \%, 50 \%, 40 \%, 30 \%, 20 \%$ to $10 \%$ were correspondingly mixed thoroughly with the river sand to obtain $100 \%$ on each sample combination. Liquid limit and plastic limit tests as well as Modified Proctor compaction were carried out on the mixture to determine the $\mathrm{OMC}$ and MDD.

\subsection{Ordinary Portland Cement Stabilization Tests.}

The cement properties and proportions used varied from $2 \%, 4 \%, 6 \%, 8 \%$ to $10 \%$ by weight of the air-dried residual soil samples. The two soil samples were deployed for the experiment. Correspondingly each sample of the residual soil varied from $98 \%, 96 \%$, $94 \%, 92 \%$ to $90 \%$ of the cement proportions. The mixture was thoroughly blended and a $6000 \mathrm{~g}$ of each was divided into five equal parts and subjected to the comparative compaction tests. Liquid limit and plastic limit tests were similarly conducted with the optimum moisture content (OMC) and maximum dry density (MDD) values obtained. 


\subsection{California Bearing Ratio Test ${ }^{8}$}

With the OMC and MDD results obtained, three specimens each of all the stabilizers were prepared for the CBR test. The river sand stabilized specimens were tested at unsoaked conditions. While one of the cement stabilized specimens was tested immediately, the remaining two were wax-cured for 6 days and thereafter soaked for 24 hours, and allowed to drain for 15 minutes. After testing in CBR machine, the average of the two readings was adopted.

\section{Presentation Of Test Results}

Table 1: Unyeghe Residual Soil and River Sand Classification - Sample No. 1

\begin{tabular}{|c|c|c|c|c|c|c|c|c|c|}
\hline $\begin{array}{l}\text { River sand } \\
\text { content } \%\end{array}$ & $\begin{array}{c}\text { MDD } \\
\mathrm{Kg} / \mathrm{m}^{3}\end{array}$ & $\begin{array}{c}\text { OMC } \\
\%\end{array}$ & $\begin{array}{c}\text { CBR } \\
\text { Unsoaked } \\
\% \\
\end{array}$ & $\mathbf{L L}$ & PL & PI & $\begin{array}{l}\text { \% passing } \\
\text { Sieve } 200\end{array}$ & \multicolumn{2}{|c|}{ Classification } \\
\hline 0 & 1950 & 11.4 & 66 & 32 & 20 & 12 & 29 & A-2 -6 & $\mathrm{SC}$ \\
\hline 10 & 2000 & 10.6 & 60 & 37 & 25 & 12 & 29 & A- $2-6$ & $\mathrm{SC}$ \\
\hline 20 & 1940 & 10.4 & 75 & 23 & 15 & 8 & 28 & A- $2-4$ & SM \\
\hline 40 & 2130 & 9.6 & 110 & 18 & NIL & NIL & 25 & A- $1-b$ & SM \\
\hline 50 & 1960 & 10.6 & 71 & 20 & NIL & NIL & 25 & $A-1-b$ & SM \\
\hline 60 & 1900 & 6.7 & 67 & 14 & NIL & NIL & 16 & $\mathrm{~A}-1-\mathrm{b}$ & SM \\
\hline 70 & 1930 & 8.3 & 83 & 18 & NIL & NIL & 16 & $A-1-b$ & SM \\
\hline
\end{tabular}

Table 2: Unyeghe Residual Soil and River Sand Classification - Sample No. 2

\begin{tabular}{|c|c|c|c|c|c|c|c|c|c|}
\hline \multirow{2}{*}{$\begin{array}{c}\text { River } \\
\text { sand } \\
\text { content } \\
\% \\
\end{array}$} & \multirow{2}{*}{$\begin{array}{l}\text { MDD } \\
\mathrm{Kg} / \mathrm{m}^{3}\end{array}$} & \multirow[t]{2}{*}{ OMC \% } & \multirow{2}{*}{$\begin{array}{c}\text { CBR } \\
\text { (Unsoaked) } \%\end{array}$} & \multirow[t]{2}{*}{$\mathbf{L L}$} & \multirow[t]{2}{*}{ PL } & \multirow[t]{2}{*}{ PI } & \multirow{2}{*}{$\begin{array}{c}\% \\
\text { passing } \\
\text { Sieve } 200\end{array}$} & \multicolumn{2}{|c|}{ Classification } \\
\hline & & & & & & & & AASHTO & USCS \\
\hline 0 & 1960 & 10.7 & 61 & 37 & 21 & 16 & 33 & A- $2-6$ & $\mathrm{SC}$ \\
\hline 10 & 1860 & 9.7 & 66 & 31 & 21 & 10 & 33 & A- $2-4$ & SM \\
\hline 20 & 1930 & 12.5 & 70 & 28 & 19 & 9 & 29 & A- $2-5$ & SM \\
\hline 30 & 2060 & 8.2 & 82 & 27 & 21 & 6 & 29 & A- $2-4$ & SM \\
\hline 40 & 1930 & 12.2 & 90 & 24 & 19 & 5 & 21 & $A-1-b$ & SM \\
\hline 50 & 2050 & 10.4 & 82 & 23 & 20 & 3 & 20 & $A-1-b$ & SM \\
\hline 60 & 2020 & 8.0 & 70 & 20 & NIL & NIL & 19 & $A-1-b$ & SM \\
\hline 70 & 1840 & 13.1 & 17 & 17 & NIL & NIL & 16 & $A-1-b$ & SM \\
\hline
\end{tabular}

Table 3: Unyeghe Residual Soil and Cement Classification - Sample No. 1

\begin{tabular}{|c|c|c|c|c|c|c|c|c|c|}
\hline \multirow{2}{*}{$\begin{array}{l}\text { Cement } \\
\text { content } \%\end{array}$} & \multirow{2}{*}{$\begin{array}{l}\mathrm{MDD} \\
\mathrm{Kg} / \mathrm{m}^{3} \\
\end{array}$} & \multirow[t]{2}{*}{ ОМС \% } & \multirow{2}{*}{$\begin{array}{c}\text { soaked } \\
\text { CBR \% } \\
\end{array}$} & \multirow[t]{2}{*}{$\mathbf{L L}$} & \multirow[t]{2}{*}{ PL } & \multirow[t]{2}{*}{ PI } & \multirow{2}{*}{$\begin{array}{l}\text { \% passing } \\
\text { Sieve } 200\end{array}$} & \multicolumn{2}{|c|}{ Classification } \\
\hline & & & & & & & & AASHTO & USCS \\
\hline 0 & 1960 & 10.7 & 26 & 37 & 21 & 16 & 33 & A- $2-4$ & SM \\
\hline 2 & 2100 & 11.2 & 70 & 28 & 20 & 8 & 40 & A- $2-4$ & SM \\
\hline 4 & 1940 & 12.3 & 81 & 28 & 21 & 7 & 41 & A- $2-4$ & SM \\
\hline 6 & 2040 & 12.9 & 87 & 27 & 22 & 5 & 42 & A- $2-4$ & SM \\
\hline 8 & 2070 & 13.2 & 95 & 17 & NIL & NIL & 43 & A- $2-4$ & SM \\
\hline 10 & 2060 & 15.1 & 110 & 18 & NIL & NIL & 44 & A- $2-4$ & SM \\
\hline
\end{tabular}

Table 4Unyeghe Residual Soil and Cement Classification - Sample No.2

\begin{tabular}{|c|c|c|c|c|c|c|c|c|c|}
\hline \multirow{2}{*}{$\begin{array}{l}\text { CEMENT } \\
\text { content \% }\end{array}$} & \multirow{2}{*}{$\begin{array}{l}\text { MDD } \\
\mathrm{Kg} / \mathrm{m}^{3}\end{array}$} & \multirow[t]{2}{*}{ ОМС \% } & \multirow{2}{*}{$\begin{array}{c}\text { SOAKED } \\
\text { CBR \% }\end{array}$} & \multirow[t]{2}{*}{$\mathbf{L L}$} & \multirow[t]{2}{*}{ PL } & \multirow[t]{2}{*}{ PI } & \multirow{2}{*}{$\begin{array}{c}\% \\
\text { passing } \\
\text { Sieve } \\
200 \\
\end{array}$} & \multicolumn{2}{|c|}{ Classification } \\
\hline & & & & & & & & AASHTO & USCS \\
\hline 0 & 1950 & 11.4 & 26 & 32 & 23 & 9 & 28 & A- $2-4$ & SM \\
\hline 2 & 2120 & 11.2 & 73 & 28 & 20 & 8 & 29 & A- $2-4$ & SM \\
\hline 4 & 2060 & 13.8 & 79 & 27 & 20 & 7 & 30 & A- $2-4$ & SM \\
\hline 6 & 2050 & 10.3 & 83 & 27 & 21 & 6 & 31 & A- $2-4$ & SM \\
\hline 8 & 2050 & 14.7 & 96 & 26 & 22 & 4 & 32 & A- $2-4$ & SM \\
\hline 10 & 2050 & 14.2 & 110 & 18 & NIL & NIL & 33 & A- $2-4$ & SM \\
\hline
\end{tabular}


Compaction Characterization and Model Prediction of Stabilized UnyegheResidual .....

Table 5: UnyegheResidual Soil Compaction at Plain Condition

\begin{tabular}{|c|c|c|c|c|}
\hline $\begin{array}{c}\text { Sample } \\
\text { No }\end{array}$ & $\begin{array}{c}\text { MDD } \\
\mathbf{K g} / \mathbf{m}^{\mathbf{3}}\end{array}$ & $\begin{array}{c}\text { NMC } \\
\mathbf{\%}\end{array}$ & $\begin{array}{c}\text { Unsoaked CBR } \\
\mathbf{\%}\end{array}$ & $\begin{array}{c}\text { Fines } \\
\mathbf{\%}\end{array}$ \\
\hline 1 & 1980 & 10.1 & 60 & 30 \\
\hline 2 & 1960 & 10.7 & 61 & 33 \\
\hline
\end{tabular}

Table 6: Comparative Compaction - Unyeghe Residual Soil and River Sand

\begin{tabular}{|c|c|c|c|}
\hline \multicolumn{4}{|c|}{ Sample Location 1} \\
\hline River Sand Content (\%) & $\begin{array}{c}\text { MDD } \\
\left(\mathrm{kg} / \mathrm{m}^{3}\right)\end{array}$ & $\begin{array}{c}\text { OMC } \\
(\%)\end{array}$ & CBR (\%) \\
\hline \multicolumn{4}{|c|}{ BS Compaction $2.5 \mathrm{~kg}-3$ Layers -61 blows } \\
\hline 10 & 1790 & 14.1 & 16 \\
\hline 20 & 1890 & 10.3 & 17 \\
\hline 30 & 1860 & 11.3 & 18 \\
\hline 40 & 1880 & 12.3 & 22 \\
\hline 50 & 1930 & 9.1 & 34 \\
\hline 60 & 1880 & 10.6 & 26 \\
\hline 70 & 1940 & 6.2 & 32 \\
\hline \multicolumn{4}{|c|}{ WAS Compaction $4.5 \mathrm{~kg}-5$ Layers -25 blows } \\
\hline 10 & 2000 & 9.2 & 32 \\
\hline 20 & 1990 & 9.8 & 34 \\
\hline 30 & 1900 & 11.8 & 36 \\
\hline 40 & 1740 & 14.6 & 38 \\
\hline 50 & 2060 & 8.6 & 39 \\
\hline 60 & 1970 & 9.8 & 43 \\
\hline 70 & 1810 & 6 & 40 \\
\hline \multicolumn{4}{|c|}{ HBS Compaction $4.5 \mathrm{~kg}-5$ Layers -61 blows } \\
\hline 10 & 1970 & 9.2 & 97 \\
\hline 20 & 2030 & 9.1 & 104 \\
\hline 30 & 2010 & 8.4 & 109 \\
\hline 40 & 2100 & 7.2 & 116 \\
\hline 50 & 2030 & 8.3 & 132 \\
\hline 60 & 2100 & 7.6 & 132 \\
\hline 70 & 2020 & 8.9 & 110 \\
\hline
\end{tabular}

Table 7: Comparative Compaction - Unyeghe Residual Soil and River Sand

\begin{tabular}{|c|c|c|c|}
\hline \multicolumn{4}{|c|}{ Sample Location 2} \\
\hline River Sand Content (\%) & $\begin{array}{c}\text { MDD } \\
\left(\mathrm{kg} / \mathrm{m}^{3}\right)\end{array}$ & OMC (\%) & CBR (\%) \\
\hline \multicolumn{4}{|c|}{ BS Compaction 2.5kg-3 Layers - 61 blows } \\
\hline 10 & 1790 & 13.6 & 14 \\
\hline 20 & 1920 & 10.5 & 15 \\
\hline 30 & 1890 & 10.4 & 16 \\
\hline 40 & 1870 & 11.5 & 19 \\
\hline 50 & 1930 & 7.5 & 31 \\
\hline 60 & 1940 & 9.7 & 32 \\
\hline 70 & 1970 & 7.1 & 25 \\
\hline \multicolumn{4}{|c|}{ WAS Compaction $4.5 \mathrm{~kg}-5$ Layers -25 blows } \\
\hline 10 & 1900 & 14 & 31 \\
\hline 20 & 1990 & 10.6 & 32 \\
\hline 30 & 1940 & 11.5 & 35 \\
\hline 40 & 1960 & 10.5 & 37 \\
\hline 50 & 1980 & 10 & 38 \\
\hline 60 & 1970 & 12.2 & 40 \\
\hline 70 & 2000 & 13.5 & 46 \\
\hline \multicolumn{4}{|c|}{ HBS Compaction $4.5 \mathrm{~kg}-5$ Layers -61 blows } \\
\hline 10 & 2040 & 8.4 & 94 \\
\hline 20 & 2040 & 8.9 & 99 \\
\hline 30 & 2080 & 6.7 & 110 \\
\hline 40 & 2040 & 6.7 & 119 \\
\hline 50 & 2010 & 9.4 & 134 \\
\hline 60 & 2000 & 8.3 & 130 \\
\hline 70 & 2050 & 6.5 & 114 \\
\hline
\end{tabular}


Compaction Characterization and Model Prediction of Stabilized UnyegheResidual .....

Table 8: Comparative Compaction - Unyeghe Residual Soil and Cement

\begin{tabular}{|c|c|c|c|}
\hline \multicolumn{3}{|c|}{ Sample Location 1 } \\
\hline Cement Content (\%) & $\begin{array}{c}\text { MDD OMC (\%) } \\
\left(\mathbf{k g} / \mathbf{m}^{\mathbf{3}}\right)\end{array}$ & Soaked CBR (\%) \\
\hline \multicolumn{3}{|c|}{ BS Compaction 2.5kg-3 Layers - 25 blows } \\
\hline 2 & 1900 & 10.4 & 80 \\
\hline 4 & 1940 & 12.3 & 98 \\
\hline 6 & 1950 & 13.1 & 14 \\
\hline 8 & 1960 & 15.2 & 118 \\
\hline 10 & 1980 & 15.8 & 124 \\
\hline 12 & 2000 & 9 & 85 \\
\hline 2 & WAS Compaction 4.5kg-5 Layers - 25 blows \\
\hline 4 & 1980 & 9.4 & 94 \\
\hline 6 & 2020 & 9.6 & 111 \\
\hline 10 & 2050 & 10.2 & 115 \\
\hline
\end{tabular}

Table 9: Comparative Compaction - Unyeghe Residual Soil and Cement

\begin{tabular}{|c|c|c|c|}
\hline \multicolumn{4}{|c|}{ Sample Location 2} \\
\hline $\begin{array}{c}\text { Cement Content } \\
(\%)\end{array}$ & $\begin{array}{c}\text { MDD } \\
\left(\mathrm{kg} / \mathrm{m}^{3}\right)\end{array}$ & $\begin{array}{c}\text { OMC } \\
(\%)\end{array}$ & $\begin{array}{c}\text { Soaked CBR } \\
(\%)\end{array}$ \\
\hline \multicolumn{4}{|c|}{ BS Compaction $2.5 \mathrm{~kg}-3$ Layers -25 blows } \\
\hline 2 & 1880 & 8.6 & 79 \\
\hline 4 & 1910 & 10 & 85 \\
\hline 6 & 1910 & 10.1 & 92 \\
\hline 8 & 1950 & 10.3 & 104 \\
\hline 10 & 1940 & 11.6 & 114 \\
\hline 12 & 1960 & 12 & 123 \\
\hline \multicolumn{4}{|c|}{ WAS Compaction $4.5 \mathrm{~kg}-5$ Layers -25 blows } \\
\hline 2 & 1980 & 10.6 & 84 \\
\hline 4 & 1900 & 10.2 & 91 \\
\hline 6 & 1910 & 9.8 & 109 \\
\hline 8 & 1930 & 9.3 & 114 \\
\hline 10 & 1960 & 8.6 & 123 \\
\hline 12 & 1970 & 8.4 & 129 \\
\hline
\end{tabular}

\section{Discussion Of Test Results}

Tables 1 to 4 present Unyeghe residual soil classification at stabilized conditions with both river sand and cement additives. Table 5 shows the natural condition of the two samples. The results of variable compactive effort on soil samples from locations 1 and 2 , treated with different river sand and cement contents are presented on Tables 6 to 9. Addition of cement increases the maximum dry density as well as the California Bearing Ratio. However, the optimum moisture content does not follow this relationship strictly. The BS compaction with river sand stabilization produced CBR values ranging from $16 \%$ to $32 \%$ and $32 \%$ to $40 \%$ with WAS compactive effort. These values are far below acceptable minimum specification [35\% and $80 \%$ ] for subbase and base course applications. HBS compaction of similar samples results in CBR values ranging from $97 \%$ to $134 \%$. Conversely BS compaction of soaked samples stabilized with cement produced CBR values from $80 \%$ to $124 \%$ and WAS compaction values ranged from $84 \%$ to $132 \%$.

\section{Multiple Nonlinear Regressed Models}

Based on analysis and utilizing multiple regressed programs ${ }^{9}$ some models were developed for Unyeghe residual soils at various levels of compaction and stabilization. These models aid prediction and optimization to determine for what values of independent variables the dependent variable is a maximum or minimum.

$\mathrm{CBR}_{\mathrm{BSr}}=4.593-.293 \mathrm{~S}-5.498 \mathrm{D}-.931 \mathrm{~W}-.572 \mathrm{C}+.007 \mathrm{~S}^{2}+.294 \mathrm{D}^{2}+.004 \mathrm{~W}^{2}+.229 \mathrm{C}^{2}-.048 \mathrm{SD}-.024 \mathrm{SW}+$

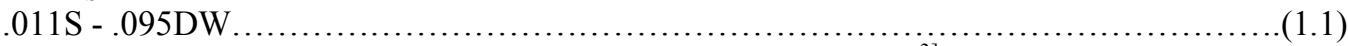

Where $\mathrm{S}=$ River sand [\%], D = Maximum dry density $\left[\mathrm{Mg} / \mathrm{m}^{3}, \mathrm{~W}=\right.$ Optimum moisture content $[\%], \mathrm{C}=$ Compactive effort $[\mathrm{kg}]$

$\mathrm{CBR}_{\mathrm{WASr}}=10.929+.437 \mathrm{~S}-4.731 \mathrm{D}+.755 \mathrm{~W}+.498 \mathrm{C}+.002 \mathrm{~S}^{2}+.248 \mathrm{D}^{2}-.325 \mathrm{~W}^{2}-.110 \mathrm{C}^{2}-.202 \mathrm{SD}-.018 \mathrm{SW}$ $+.097 \mathrm{SC}-.091 \mathrm{DW}$.

Where $\mathrm{S}=$ River sand $[\%], \mathrm{D}=$ Maximum dry density $[\mathrm{Mg} / \mathrm{g}], \mathrm{W}=$ Optimum moisture content $[\%], \mathrm{C}=$ Compactive effort $[\mathrm{kg}]$ 
$\mathrm{CBR}_{\mathrm{HBSr}}=54.889-.635 \mathrm{~S}-3.744 \mathrm{D}-1.930 \mathrm{~W}-.126 \mathrm{C}+.001 \mathrm{~S}^{2}+.780 \mathrm{D}^{2}-.459 \mathrm{~W}^{2}+.281 \mathrm{C}^{2}+.253 \mathrm{SD}+$

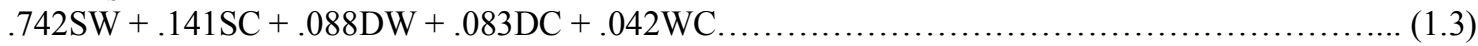

Where $\mathrm{S}=$ River sand [\%], $\mathrm{D}=$ Maximum dry density $[\mathrm{Mg} / \mathrm{g}], \mathrm{W}=$ Optimum moisture content [\%], $\mathrm{C}=$ Compactive effort $[\mathrm{kg}]$

$\mathrm{CBR}_{\mathrm{BSc}}=16.983+5.820 \mathrm{~N}+2.112 \mathrm{D}+4.563 \mathrm{~W}-.162 \mathrm{C}+.137 \mathrm{~N}^{2}+.366 \mathrm{D}^{2}-.387 \mathrm{~W}^{2}-.648 \mathrm{C}^{2}+.117 \mathrm{ND}+$

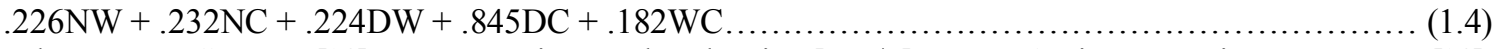

Where $\mathrm{N}=$ Cement $[\%], \mathrm{D}=$ Maximum dry density $[\mathrm{Mg} / \mathrm{g}], \mathrm{W}=$ Optimum moisture content $[\%], \mathrm{C}=$ Compactive effort $[\mathrm{kg}]$

$\mathrm{CBR}_{\mathrm{WASc}}=34.430-2.652 \mathrm{~N}-1.728 \mathrm{D}-1.984 \mathrm{~W}+.226 \mathrm{C}+.336 \mathrm{~N}^{2}+.168 \mathrm{D}^{2}-.422 \mathrm{~W}^{2}-.503 \mathrm{C}^{2}-.241 \mathrm{ND}+$

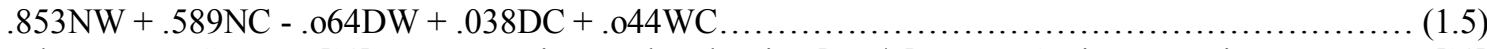

Where $\mathrm{N}=$ Cement $[\%], \mathrm{D}=$ Maximum dry density $[\mathrm{Mg} / \mathrm{g}], \mathrm{W}=$ Optimum moisture content $[\%], \mathrm{C}=$ Compactive effort $[\mathrm{kg}]$

Table10: Multiple Regressed Variables for Measured and Computed CBR Values (Residual Soil and River Sand Stabilization)

\begin{tabular}{|c|c|c|c|c|c|}
\hline \multicolumn{6}{|c|}{ Sample location 1} \\
\hline $\begin{array}{l}\text { River Sand } \\
\text { Content (\%) }\end{array}$ & $\begin{array}{c}\text { Compactive Effort } \\
\text { (Kg) }\end{array}$ & $\underset{\left(\mathrm{kg} / \mathrm{m}^{3}\right)}{\operatorname{MDD}}$ & $\begin{array}{c}\text { OMC } \\
(\%)\end{array}$ & $\begin{array}{c}\text { Measured CBR } \\
(\%)\end{array}$ & Computed CBR (\%) \\
\hline \multicolumn{6}{|c|}{ BS Compaction $2.5 \mathrm{~kg}-3$ Layers -61 blows } \\
\hline 10 & 2.5 & 1.79 & 14.1 & 16 & 0.310 \\
\hline 20 & 2.5 & 1.89 & 10.3 & 17 & 7.473 \\
\hline 30 & 2.5 & 1.86 & 11.3 & 18 & 8.932 \\
\hline 40 & 2.5 & 1.88 & 12.3 & 22 & 11.529 \\
\hline 50 & 2.5 & 1.93 & 9.1 & 34 & 24.464 \\
\hline 60 & 2.5 & 1.88 & 10.6 & 26 & 28.409 \\
\hline 70 & 2.5 & 1.94 & 6.2 & 32 & 49.406 \\
\hline \multicolumn{6}{|c|}{ WAS Compaction $4.5 \mathrm{~kg}-5$ Layers -25 blows } \\
\hline 10 & 4.5 & 2 & 9.2 & 32 & 54.888 \\
\hline 20 & 4.5 & 1.99 & 9.8 & 34 & 73.189 \\
\hline 30 & 4.5 & 1.9 & 11.8 & 36 & 100.994 \\
\hline 40 & 4.5 & 1.74 & 14.6 & 38 & 138.552 \\
\hline 50 & 4.5 & 2.06 & 8.6 & 39 & 114.867 \\
\hline 60 & 4.5 & 1.97 & 9.8 & 43 & 137.326 \\
\hline 70 & 4.5 & 1.81 & 6 & 40 & 130.571 \\
\hline \multicolumn{6}{|c|}{ HBS Compaction 4.5kg-5 Layers -61 blows } \\
\hline 10 & 4.5 & 1.97 & 9.2 & 97 & 197.255 \\
\hline 20 & 4.5 & 2.03 & 9.1 & 104 & 282.396 \\
\hline 30 & 4.5 & 2.01 & 8.4 & 109 & 346.554 \\
\hline 40 & 4.5 & 2.1 & 7.2 & 116 & 385.247 \\
\hline 50 & 4.5 & 2.03 & 8.3 & 132 & 504.436 \\
\hline 60 & 4.5 & 2.1 & 7.6 & 132 & 550.686 \\
\hline 70 & 4.5 & 2.02 & 8.9 & 110 & 701.278 \\
\hline
\end{tabular}

Table11: Multiple Regressed Variables for Measured and Computed CBR Values (Residual Soil and River Sand Stabilization)

\begin{tabular}{|c|c|c|c|c|c|}
\hline \multicolumn{6}{|c|}{ Sample Location 2} \\
\hline River Sand Content (\%) & $\begin{array}{l}\text { Compactive } \\
\text { Effort }(\mathrm{Kg})\end{array}$ & $\begin{array}{c}\text { MDD } \\
\left(\mathrm{kg} / \mathrm{m}^{3}\right)\end{array}$ & $\begin{array}{c}\text { OMC } \\
(\%)\end{array}$ & $\begin{array}{c}\text { Measured CBR } \\
(\%)\end{array}$ & $\begin{array}{c}\text { Computed CBR } \\
(\%)\end{array}$ \\
\hline \multicolumn{6}{|c|}{ BS Compaction $2.5 \mathrm{~kg}-3$ Layers -61 blows } \\
\hline 10 & 2.5 & 1.79 & 13.6 & 14 & 0.925 \\
\hline 20 & 2.5 & 1.92 & 10.5 & 15 & 7.311 \\
\hline 30 & 2.5 & 1.89 & 10.4 & 16 & 10.624 \\
\hline 40 & 2.5 & 1.87 & 11.5 & 19 & 13.073 \\
\hline 50 & 2.5 & 1.93 & 7.5 & 31 & 28.061 \\
\hline 60 & 2.5 & 1.94 & 9.7 & 32 & 30.800 \\
\hline 70 & 2.5 & 1.97 & 7.1 & 25 & 47.017 \\
\hline \multicolumn{6}{|c|}{ WAS Compaction $4.5 \mathrm{~kg}-5$ Layers -25 blows } \\
\hline 10 & 4.5 & 1.9 & 14 & 31 & 86.802 \\
\hline 20 & 4.5 & 1.99 & 10.6 & 32 & 78.032 \\
\hline 30 & 4.5 & 1.94 & 11.5 & 35 & 99.267 \\
\hline 40 & 4.5 & 1.96 & 10.5 & 37 & 108.709 \\
\hline 50 & 4.5 & 1.98 & 10 & 38 & 122.076 \\
\hline 60 & 4.5 & 1.97 & 12.2 & 40 & 154.835 \\
\hline 70 & 4.5 & 2 & 13.5 & 46 & 184.186 \\
\hline
\end{tabular}


Compaction Characterization and Model Prediction of Stabilized UnyegheResidual .....

\begin{tabular}{|c|c|c|c|c|c|}
\hline \multicolumn{7}{|c|}{ HBS Compaction 4.5kg-5 Layers-61 blows } \\
\hline 10 & 4.5 & 2.04 & 8.4 & 94 & 186.056 \\
\hline 20 & 4.5 & 2.04 & 8.9 & 99 & 277.965 \\
\hline 30 & 4.5 & 2.08 & 6.7 & 110 & 298.314 \\
\hline 40 & 4.5 & 2.04 & 6.7 & 119 & 365.750 \\
\hline 50 & 4.5 & 2.01 & 9.4 & 134 & 553.685 \\
\hline 60 & 4.5 & 2 & 8.3 & 130 & 584.058 \\
\hline 70 & 4.5 & 2.05 & 6.5 & 114 & 560.454 \\
\hline
\end{tabular}

- Series1 Linear (Series1)

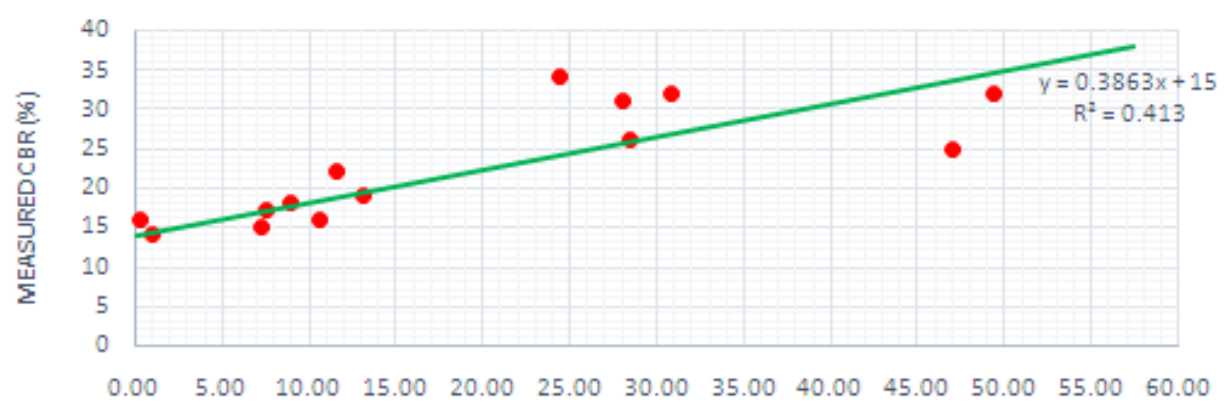

Fig 1: Cross Plot of measured vs Computed CBR Values using Equationl.1

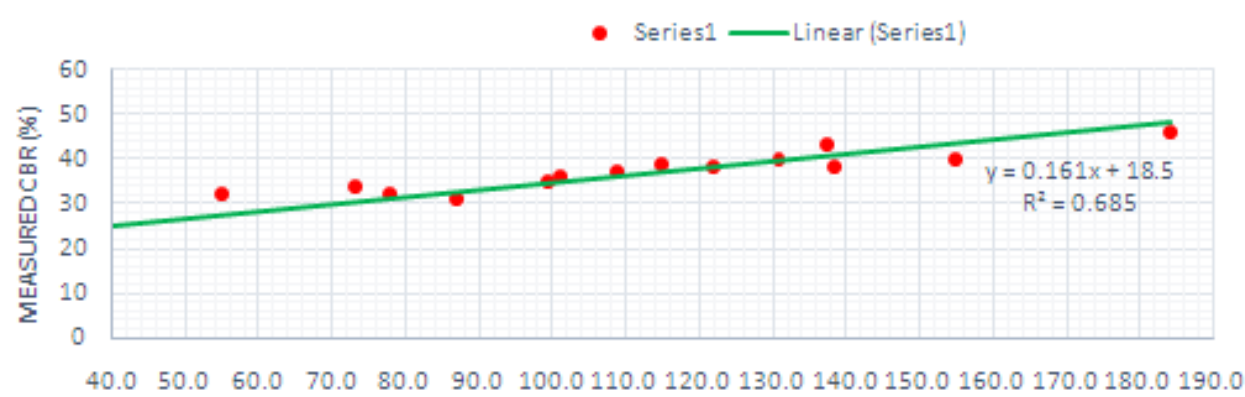

Fig. 2: Cross Plot of Measured vs Computed CBR Values using Equation 1.2

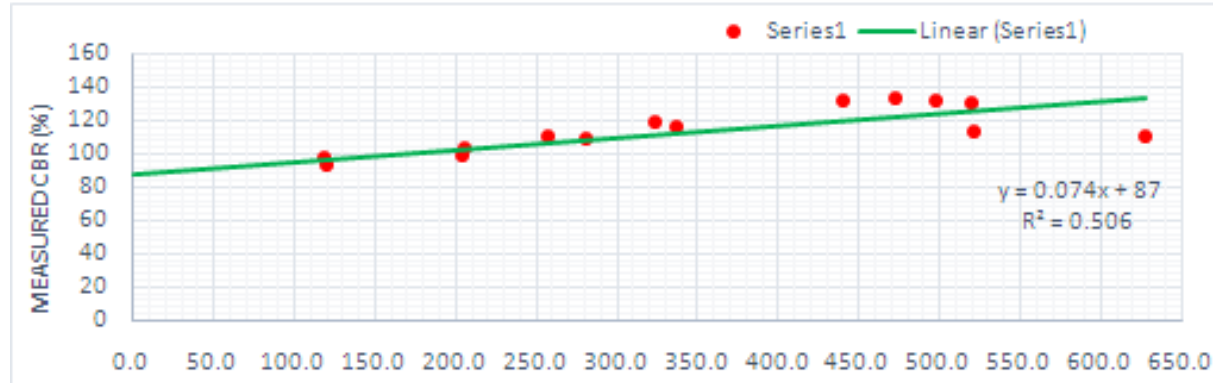

Fig. 3: Cross Plot of Measured vs Computed CBR Values using Equation 1.3

Table12 Multiple Regressed Variables for Measured and Computed CBR Values (Residual Soil and Cement Stabilization)

\begin{tabular}{|c|c|c|c|c|c|}
\hline \multicolumn{7}{|c|}{ Sample Location 1 } \\
\hline Cement Content (\%) & $\begin{array}{c}\text { Compactive Effort } \\
(\mathbf{K g})\end{array}$ & $\begin{array}{c}\text { MDD } \\
\left(\mathbf{k g} / \mathbf{m}^{\mathbf{3}}\right)\end{array}$ & $\begin{array}{c}\text { OMC } \\
\mathbf{( \% )}\end{array}$ & $\begin{array}{c}\text { Measured } \\
\text { CBR (\%) }\end{array}$ & Computed CBR (\%) \\
\hline \multicolumn{7}{|c|}{ BS Compaction 2.5kg-3 Layers - 25 blows } \\
\hline 2 & 2.5 & 1.9 & 10.4 & 80 & 55.520 \\
\hline 4 & 2.5 & 1.94 & 12.3 & 88 & 70.915 \\
\hline 6 & 2.5 & 1.95 & 13.1 & 97 & 90.178 \\
\hline 8 & 2.5 & 1.96 & 14 & 105 & 110.424 \\
\hline 10 & 2.5 & 1.98 & 15.2 & 118 & 130.901 \\
\hline
\end{tabular}


Compaction Characterization and Model Prediction of Stabilized UnyegheResidual .....

\begin{tabular}{|c|c|c|c|c|c|}
\cline { 3 - 5 } 12 & 2.5 & 2 & 15.8 & 124 & 155.011 \\
\hline \multicolumn{7}{|c|}{ WAS Compaction 4.5kg-5 Layers - 25 blows } \\
\hline 2 & 4.5 & 1.98 & 9 & 85 & 93.371 \\
\hline 4 & 4.5 & 2.02 & 9.4 & 94 & 126.154 \\
\hline 6 & 4.5 & 2.05 & 9.6 & 111 & 160.801 \\
\hline 8 & 4.5 & 2.06 & 10.2 & 115 & 204.161 \\
\hline 10 & 4.5 & 2.05 & 11.6 & 125 & 265.413 \\
\hline 12 & 4.5 & 2.06 & 13.4 & 132 & 343.555 \\
\hline
\end{tabular}

Table13Multiple Regressed Variables for Measured and Computed CBR Values (Residual Soil and Cement Stabilization)

\begin{tabular}{|c|c|c|c|c|c|}
\hline \multicolumn{6}{|c|}{ Sample Location 2} \\
\hline $\begin{array}{c}\text { Cement Content } \\
(\%)\end{array}$ & $\begin{array}{c}\text { CompactiveEffort } \\
\text { (Kg) }\end{array}$ & $\begin{array}{c}\text { MDD } \\
\left(\mathrm{Kg} / \mathrm{m}^{3}\right)\end{array}$ & $\begin{array}{c}\text { OMC } \\
(\%)\end{array}$ & $\begin{array}{l}\text { Measured } \\
\text { CBR (\%) }\end{array}$ & Computed CBR (\%) \\
\hline \multicolumn{6}{|c|}{ BS Compaction 2.5kg-3 Layers -25 blows } \\
\hline 2 & 2.5 & 1.88 & 8.6 & 79 & 57.916 \\
\hline 4 & 2.5 & 1.91 & 10 & 85 & 75.798 \\
\hline 6 & 2.5 & 1.91 & 10.1 & 92 & 96.211 \\
\hline 8 & 2.5 & 1.95 & 10.3 & 104 & 118.102 \\
\hline 10 & 2.5 & 1.94 & 11.6 & 114 & 139.912 \\
\hline 12 & 2.5 & 1.96 & 12 & 123 & 164.265 \\
\hline \multicolumn{6}{|c|}{ WAS Compaction $4.5 \mathrm{~kg}-5$ Layers -25 blows } \\
\hline 2 & 4.5 & 1.98 & 10.6 & 84 & 106.274 \\
\hline 4 & 4.5 & 1.9 & 10.2 & 91 & 133.855 \\
\hline 6 & 4.5 & 1.91 & 9.8 & 109 & 163.010 \\
\hline 8 & 4.5 & 1.93 & 9.3 & 114 & 192.333 \\
\hline 10 & 4.5 & 1.96 & 8.6 & 123 & 220.036 \\
\hline 12 & 4.5 & 1.97 & 8.4 & 129 & 256.043 \\
\hline
\end{tabular}

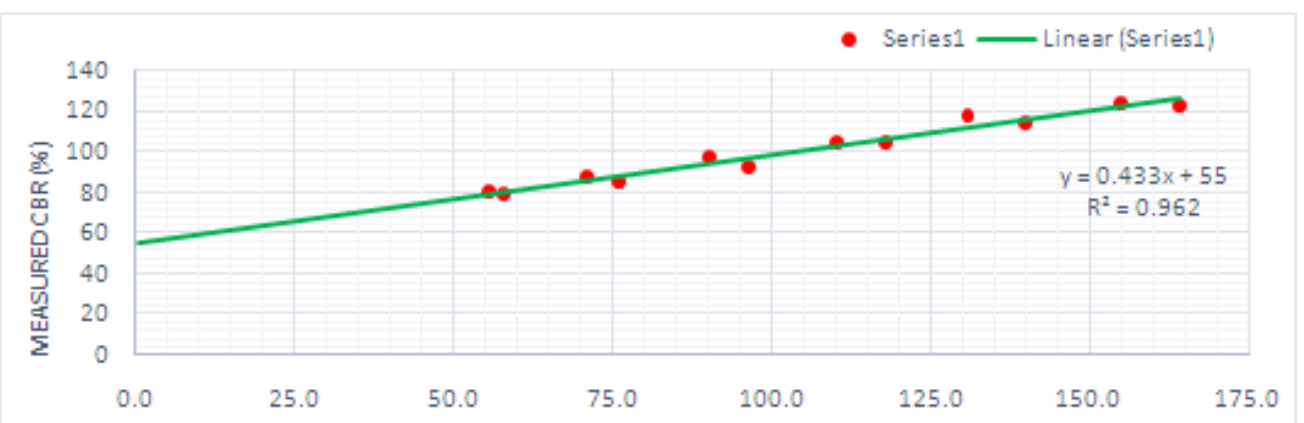

Fig. 4: Cross Plot of Measured is Computed CBR Values using Equation 1.4

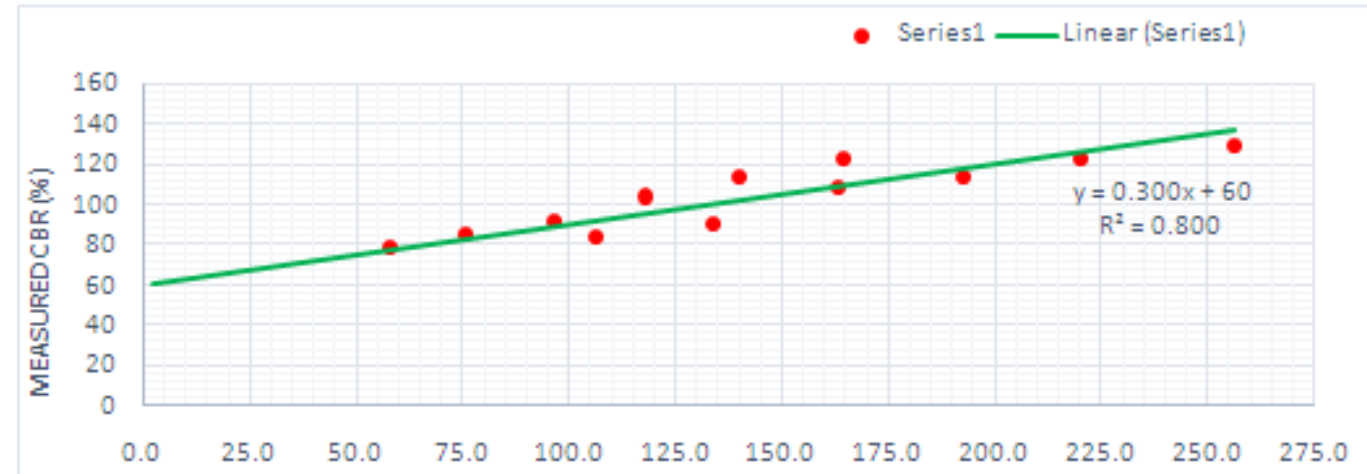

Fig. 5: Cross Plot of Measured vs Computed CBR Values using Equation 1.5

\section{Conclusion}

Equations 1.2 and 1.3 revealed that with $30 \%$ river sand content to dry weight of Unyeghe residual soil undergoing WAS or HBS compaction, CBR values of $100 \%$ and $346 \%$ could be obtained thus confirming the superiority of HBS compaction as a foremost parameter in CBR optimization. 
However equations 1.4 and 1.5 deal with the soaked material specification with a minimum acceptable CBR limit of $80 \%$. With $6 \%$ cement content the derived CBR values for both BS and WAS compaction are $96 \%$ and $163 \%$ respectively while the measured CBR values are $92 \%$ and $109 \%$. The economic viability of cement stabilization is a subject for comparative cost analysis.

The accuracy and reliability of the models $1.1-1.5$ were checked by comparing the computed and measured values of the California Bearing Ratio - CBR and computing the correlation coefficients. Figures I to $\mathrm{V}$ illustrate the computed and measured values of CBR based on the nonlinear regressed models. The straight lines in the figures represent the lines of perfect equality where the values being compared are exactly equal.

The cross correlations of the CBR parameters are designed to examine the significance and compatibility of measured and computed values of the models.

The correlation coefficients $\mathrm{R}^{2}$ at $95 \%$ confidence interval are $.413, .6852$, and .5066 for CBR with river sand content at $10 \%-70 \%$ while that for cement content at $2 \%-10 \%$ are .9624 , and .8602 . These values are statistically significant and therefore suggest that the measured and computed values of CBR are comparable.

\section{Acknowledgement}

The author would like to acknowledge the contribution of Esudo Engineering Ventures for support rendered in the course of this research.

\section{References}

[1]. Garber, N.J. and Hoel, L.A. [1999] "Soil Engineering For Highway Design” 817-866 Fe Federal Ministry of Works \& Housing, General Specifications (Roads and Bridges), vol. 11, 1997. deral Ministry of Works \& Housing, General Specifications (Roads and Bridges), vol. 11, 1997.

[2]. Budhu, M. [1999] Soil Mechanics And Foundations. John Wiley \& Sons Inc. N.Y., U.S.A.

[3]. Al-Aghbari, M.Y. and Dutta, R.K. [2005] Suitability of Desert Sand Cement Mixes for Base Courses in Highway Pavements. Electronic Journal of Geotechnical Engineering, Vol. 10, 2005, Bundle D.

[4]. Casagrande, L. [1952] "Electro-Osmotic Stabilization of Soils" Journal, Boston Society of Civil Engineers, vol.39, p51.

[5]. Deboucha, S., Hashim, R. and Alwi, A. [2008] "Engineering Properties of Stabilized Tropical Peat Soils" Electronic Journal of Geotechnical Engineering.

[6]. Omotosho, P. O., [2004] "Effects of Stabilisation on the Performance of Deltaic Lateritic Soils as a Road Pavement Material" $\mathrm{Ph} . \mathrm{D}$ Thesis, University of Nigeria, Nsukka, Nigeria.

[7]. American Association of State Highway and Transportation Officials test T 193

[8]. Erwin K, [2004] Advanced Engineering Mathematics $8^{\text {th }}$ Edition, John Wiley \& Sons, Inc. 